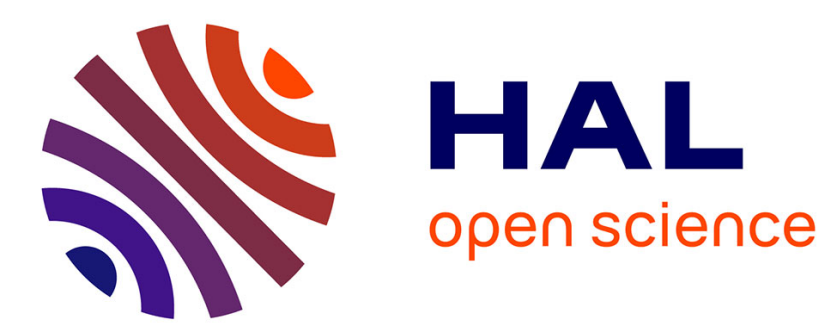

\title{
AGING EFFECTS ON THE RELAXATION OF TORQUE AND MAGNETIZATION IN A CANONICAL SPIN GLASS
}

C. Giovannella, L. Fruchter, I. Campbell

\section{> To cite this version:}

C. Giovannella, L. Fruchter, I. Campbell. AGING EFFECTS ON THE RELAXATION OF TORQUE AND MAGNETIZATION IN A CANONICAL SPIN GLASS. Journal de Physique Colloques, 1988, 49 (C8), pp.C8-1043-C8-1044. 10.1051/jphyscol:19888476 . jpa-00228678

\section{HAL Id: jpa-00228678 https://hal.science/jpa-00228678}

Submitted on 1 Jan 1988

HAL is a multi-disciplinary open access archive for the deposit and dissemination of scientific research documents, whether they are published or not. The documents may come from teaching and research institutions in France or abroad, or from public or private research centers.
L'archive ouverte pluridisciplinaire HAL, est destinée au dépôt et à la diffusion de documents scientifiques de niveau recherche, publiés ou non, émanant des établissements d'enseignement et de recherche français ou étrangers, des laboratoires publics ou privés. 


\title{
AGING EFFECTS ON THE RELAXATION OF TORQUE AND MAGNETIZATION IN A CANONICAL SPIN GLASS
}

\author{
C. Giovannella ${ }^{1}$, L. Fruchter and I. A. Campbell \\ Physique des Solides, 91405 Orsay, France
}

\begin{abstract}
Using a capacity cell torquemeter we have investigated the aging effects on the form of the torque relaxation in a CuMn $5 \%$ spin glass. The data show that in moderate fields the spin glass evolves strongly with waiting time after field change if prepared by zero field cooling (ZFC) followed by an applied field, or if measurements are performed after some other variation of the magnetic field strength. After cooling in constant field (FC) the relaxation rate does not depend on the waiting time after cooling.
\end{abstract}

The evolution in time of glassy systems is characterized by a slow relaxation of the relevant physical quantities. In particular in spin glasses (SG) the approach to equilibrium is via a slow and non exponential relaxation of the remanent magnetization [1]. The broad distribution of effective characteristic relaxation times $\tau$ mirrors that of the ranges of times needed by the system to explore the complex available phase space. This situation is often presented in terms of overcoming a range of free energy barriers. It is important to distinguish between two relaxation phenomena-relaxation after a change in the external feld, and the waiting time effect following a change in temperature which shows up as a change in the relaxation per se observed after a subsequent change of field. Indeed aging effects on relaxation dynamics, and also changes induced by a variation of the field and of the temperature, have been intensively investigated by studying the evolution of the shape of the remanent magnetization decay as a function of the parameters $t_{\mathrm{w}}, H$ and $T[2,3]$.

Recently it has been shown that complementary information on the dynamics of the spin glasses can be obtained by studying the decay of the magnetic torque intensity [4]. In previous papers we discussed the implications of the torque decay observations on the relaxation models for SG and the influence of the parameter $T$ on the relaxation towards the equilibrium of a SG prepared initially in different states by following different experimental protocols. Here we will describe the effects induced by waiting times on the shape of the torque relaxation of a canonical CuMn $5 \%$ SG $\left(T_{\mathrm{g}} \approx 27.5 \mathrm{~K}\right)$. For the torque experiments there are two types of waiting times: a wait after a temperature change (as in the magnetization experiments) and a wait after a change in the strength of the field, before the field is turned. For an isotropic sample the torque signal is zero until field turning. Our results can be compared with those obtained by the study of the magnetization decay for low and high field experiments $[2,3,5]$.

The data, some of which are presented in the figures, have been obtained following four different ex- perimental protocols: 1) $\mathrm{FC} \theta$ : the sample is first field cooled (FC) under field $H$ to temperature $T$, then after a waiting time $t_{\mathrm{w}}$, the field is rotated to an angle $\theta$ with respect to the cooling direction (typically we used $\theta=5^{\circ}$ ). The torque intensity $\Gamma$ is monitored continuously; 2) ZFC $\theta$ : after zero field cooling (ZFC), the field is increased to a value $H$ and, after a waiting time $t_{\mathrm{w}}^{*}$ in field, is rotated through $\theta ; 3) \mathrm{FC} \Downarrow h \theta:$ after FC in field $H$, the field is reduced to a value $h$ (typically $1 \mathrm{kG})$ and, after $t_{\mathrm{w}}^{*}$, is rotated through $\theta$; 4) $\mathrm{FC}(\sigma)$ : after $\mathrm{FC}$ the field is reduced to a low value $(50 \mathrm{G})$ and after $t_{\mathrm{w}}^{*}$, the magnet is turned through $90^{\circ}$. The useful time window is from 1 to 2000 seconds. We recall that in the protocol $\mathrm{FC}(\sigma)$ we are in the weak field limit [4], i.e. we are measuring the decay of the remanent magnetization $(\Phi \approx \sigma)$.

Figure 1 shows in a $\ln \left(-\ln \left(\Gamma / \Gamma_{0}\right)\right)-\ln t$ plot a torque relaxation recorded at $4.2 \mathrm{~K}$ after protocol 1 with $H=8.7 \mathrm{kG}$ and $\theta=5^{\circ}$; the data have been normalized to their initial value $\Gamma_{0}$. A straight line on this particular plot would indicate a stretched exponential decay $\left(\exp -(t / \tau)^{\beta}\right)$, which is indeed the behaviour observed for $\ln (t)>3$. The origin, $t=0$, where we measured the initial torque $\Gamma_{0}$ is defined to better than 2 seconds. The uncertainty in the origin slightly affects the form of the decay but in the short time range only. Under this protocol the form of $\Gamma(t)$ for a given $H$ is independent of the waiting time after cooling $t_{\mathrm{w}}$ within the precision of our experiment

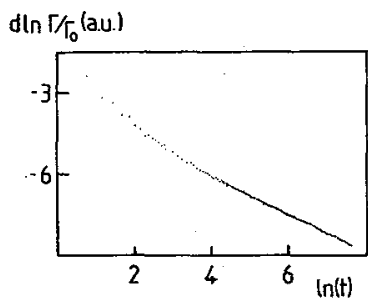

Fig. 1. - Plot of $\ln \left(-\ln \left(\Gamma / \Gamma_{0}\right)\right)$ (indicated as d ln $\left(\Gamma / \Gamma_{0}\right)$ ) against ln $(t)$ for a CuMn 5 at $\%$ sample after field cooling. $T=4.2 \mathrm{~K}, H=8.7 \mathrm{kG}, \theta=5^{\circ} . \Gamma$ is the torque and $\Gamma_{0}$ the initial value immediately after turning.

\footnotetext{
${ }^{1}$ Permanent äddress: Dip. di Fisica, Roma II, Via O. Raimondo, 00173 Roma, Italy.
} 
and in our experimental time window. The form of the curve did not depend on the angle $\theta$ either, for small or moderate turning angles, which suggests that we are observing the limiting transverse response as $\theta$ tends to zero.

Figure 2 shows a set of torque relaxations recorded after protocols 2,3 and 4 for different $t_{\mathrm{w}}^{*}: 0,30^{\prime}, 2 \mathrm{~h}$. The parameters used in the experiments are given in the figure captions. The data is presented on a ln-ln scale. A straight line in this plot would imply a power law decay $\left(t^{-\alpha}\right)$. The relaxation observed after the different protocols did not depend on the waiting time at fixed field after cooling, but clearly did depend on the waiting time after field strength change before the field was turned.

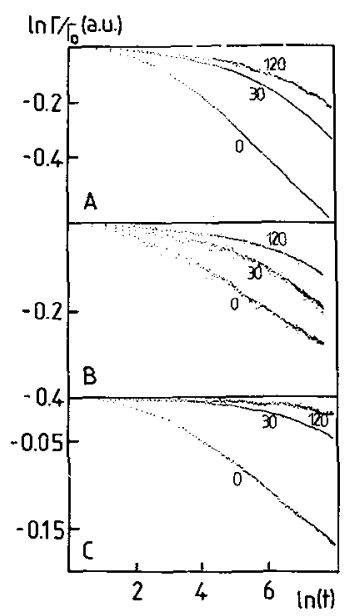

Fig. 2. - Plot of $\ln \left(\Gamma / \Gamma_{0}\right)$ against $\ln (t)$ for: A) ZFC $\theta$ with the same $T, H, \theta$ as figure 1 but with waiting times after raising the field of 0,30 and 120 minutes; $B$ ) protocol 3 , sarne initial conditions, turning field $1 \mathrm{kG}$, waiting times before turning 0,30 and 120 minutes; $C$ ) protocol 4 , same initial conditions, turning field $50 \mathrm{G}$, same waiting times.

From the ensemble of the data we find that in our experimental time window the shape of the relaxation cannot be represented by any simple and unique law over the whole time range.

For our range of fields, the waiting time after cooling for fixed field strength does not affect subsequent relaxation when the field is turned. On the other hand when there is a waiting time between the moment the field is applied after ZFC and the moment it is turned, there is a clear evolution in the shape of the torque relaxation towards that observed after the FC $\theta$ protocol for the same $H$ and $T$. The longer the waiting time in field $t_{\mathrm{w}}^{*}$, the slower the subsequent relaxation. After a waiting time of two hours before turning, the relaxation curve is within $1 \%$ of the $\mathrm{FC} \theta$ curve (not shown in the figure). Similarly, when the field is reduced before turning, protocols 3 and 4 , the relaxation depends on the waiting time before turning.
A small turning angle torque experiment can be considered as a measurement of the transverse response to a small transverse field; waiting time effects imply reorganizations of the spin system which are only "revealed" after a subsequent operation. In magnetization experiments, waiting time effects after cooling have been observed for small applied fields $[2,3]$ but they tend to be suppressed at larger fields [6]. The fact that we see no waiting time effects after FC if the strength of the field is kept constant is consistent with this. We can deduce that cooling in a fairly strong field puts the system into a well defined state and there is no internal reorganization during a waiting period at fixed field strength and direction. When the field is then turned slightly the system is out of equilibrium due to competition between exchange and anisotropy terms [4] and it relaxes towards a new equilibrium. After ZFC and application of $H$, there is a strong internal reorganization before the field is turned which appears as magnetization relaxation or as waiting time effects in the torque. There are large quantitative differences however between the two observed effects. In similar experimental conditions to ours (CuMn $5 \%$ sample, ZFC and then $10 \mathrm{kG}$ applied field) Omari et al. (5) observed changes in magnetization $M$ at $4 \mathrm{~K}$ of only about $2 \%$ for the period between 1 minute and 1 hour after putting on the field. We observe almost no waiting time effect on the initial torque signal $\Gamma_{0}$ immediately after turning (which is consistent with the $M$ data as $\Gamma_{0}$ is proportional to $M$ ), but we see, figure 2 , spectacular changes in the torque relaxation pattern as a function of $t_{\mathrm{w}}^{*}$. This is evidence that the system is undergoing an internal reorganization during the waiting time in field; this reorganization hardly changes the value of $M$ but it does drastically effect the torque relaxation. Like the standard waiting time effect $[2,3]$ torque relaxation is a method of investigating internal reorganization processes which take place with little effect on $M$; this technique gives complementary information on the reorganization in the presence of strong applied fields.

[1] Ngai, K. L., Comments Sol. St Phys. 9 (1979) 127.

[2] Svedlindh, P., Granberg, P., Nordblad, P., Lundgren, L. and Chen, H. S., Phys. Rev. B 35 (1987) 268.

[3] Alba, M., Ocio, M. and Hammann, J., Europhys. Lett. 2 (1986) 45.

[4] Giovannella, C., Campbell, I. A. and Hippert, F., Phys. Rev. Lett. 59 (1987) 1031.

[5] Omari, R., Préjean, J. J. and Souletie, J., J. Phys. France 45 (1984) 1809.

[6] Alba, M., Vincent, E., Hammann, J. and Ocio, M., J. Appl. Phys. 61 (1987) 4092. 